\section{Mehr Komplettremissionen mit neoadjuvantem Bevacizumab bei HER2-negativem Brustkrebs}

\author{
In der ARTemis-Studie sollten Wirksamkeit und Verträglichkeit von \\ Bevacizumab zusätzlich zu einer neoadjuvanten Standardchemotherapie \\ bei HER2-negativem frühem Mammakarzinom geprüft werden.
}

\begin{abstract}
nsgesamt 800 Frauen mit frühem invasivem HER2-negativem Mammakarzinom wurden in die Phase-III-Studie aufgenommen (Tumorgröße $>20 \mathrm{~mm}$, jeder Nodalstatus). Die Patientinnen erhielten randomisiert entweder 3 Zyklen D-FEC allein (Docetaxel, Fluorouracil, Epirubicin, Cyclophosphamid) oder zusätzlich Bevacizumab (Bev + D-FEC). Im experimentellen Arm erhielten die Patientinnen zusätzlich 4 Zyklen Bevacizumab. Primärer Endpunkt der Studie war das pathologisch komplette Ansprechen (pCR).

Daten von 781 Patientinnen konnten für die Analyse des primären Endpunkts ausgewertet werden. Insgesamt zeigte sich, dass die pCR abhängig war vom ERStatus (ER-negativ 38\% mit pCR, schwach positiv $41 \%$, stark positiv $7 \%$;
\end{abstract}

$\mathrm{p}<0,0001)$. Auch der Tumorgrad spielte eine Rolle (Grad 1/2: $7 \%$ pCR, Grad 3: $29 \%$ pCR; $\mathrm{p}<0,0001)$.

Die Behandlung mit Bev + D-FEC führte zu einer signifikant höheren Rate an pCR als mit D-FEC allein (22 vs. $17 \%$; $\mathrm{p}=0,03)$. In der Bev + D-FEC-Gruppe konnten $48 \%$ der Patientinnen brusterhaltend operiert werden, im Kontroll$\operatorname{arm} 51 \%(p=0,47)$. In der Gesamtpopulation benötigten Frauen mit pCR seltener eine Mastektomie als solche ohne pCR ( 29 vs. $54 \%$; p < 0,0001).

Grad-3/4-Toxizitäten wurden in der erwarteten Häufigkeit in beiden Studienarmen beobachtet. Allerdings hatten mehr Patientinnen in der Bev + D-FEC-Gruppe eine Grad-4-Neutropenie (22 vs. 17\%).

Die Gabe von 4 Zyklen Bevacizumab zusätzlich zu D-FEC bei HER2-negati- vem frühem Brustkrebs verbesserte somit signifikant die pCR-Rate. Es ist jedoch noch offen, ob dies auch zu einem verbesserten krankheitsfreien (DFS) und Gesamtüberleben (OS) führt.

Wahrscheinlich, so die Forscher, lasse sich erst durch Metaanalysen verschiedener vergleichbarer neoadjuvanter Studien Subgruppen ermitteln, die nach einer erfolgreichen neoadjuvanten Therapie mit Bevacizumab einen klinischen Langzeitnutzen in Form eines besseren DFS und OS haben.

Fazit: Die Addition von 4 Zyklen Bevacizumab zu einer Therapie mit D-FEC bei HER2-negativem frühem Brustkrebs erhöhte signifikant die Rate pathologisch kompletter Remissionen. Die Auswirkungen auf den klinischen Langzeitverlauf können erst nach einer längeren Nachbeobachtung beurteilt werden.

Brigitte Schalhorn

Earl HM et al. Efficacy of neoadjuvant bevacizumab added to docetaxel followed by fluorouracil, epirubicin, and cyclophosphamide, for women with HER2-negative early breast cancer (ARTemis): an open-label, randomised, phase 3 trial. Lancet Oncol. 2015;16(6):656-66.

\section{HER2-positiver Brustkrebs: weiterer Nutzen von Everolimus in der Erstlinie}

Da der mTOR-Inhibitor Everolimus resistente Tumoren sensitiv gegen Trastuzumab machen kann, wurde in der BOLERO-1-Studie untersucht, ob er in der Ersttherapie bei Patientinnen mit fortgeschrittenem HER2-positivem Mammakarzinom die Effektivität von Trastuzumab und Paclitaxel steigert.
HR einer Hormontherapie (23 bzw. $25 \%)$ unterzogen.

Nach einer medianen Nachbeobachtung von gut 3 Jahren unterschied sich das mediane progressionsfreie Überleben (PFS) der Patientinnen in beiden Behandlungsarmen nicht (14,95 vs. 14,49 Monate; HR 0,89; $p=0,1166)$. Auch die Remissionsraten (CR und PR) lagen mit Werten zwischen 67 und $73 \%$ im gleichen Bereich. In der Subgruppe der HRnegativen Patientinnen verlängerte die Addition von Everolimus aber das mediane PFS von 13,08 auf 20,27 Monate (HR 0,66). Dabei überschritt der p-Wert mit
0,0049 jedoch die vorher festgelegte Signifikanzgrenze $(0,0044)$.

Durch die Addition von Everolimus wurde die Therapie deutlich toxischer. Dies betraf insbesondere Stomatitis (Grad 1/2: 67 vs. $32 \%$, Grad 3/4: 13 vs. $1 \%)$ und Diarrhö (Grad 1/2: 57 vs. $47 \%$, Grad 3/4: 9 vs. $4 \%$ ). Aber auch Grad-3/4Neutropenien (25 vs. $15 \%$ ) und Grad-3/4Anämien (10 vs. $3 \%$ ) waren häufiger. 17 Patienten starben in der Ev-TP-Gruppe nebenwirkungsbedingt.

Fazit: Die Gesamtkohorte profitierte nicht von Everolimus, möglicherweise aber die Subgruppe der HR-negativen Patientinnen. Bei diesen Frauen sollte der Stellenwert von Everolimus weiter untersucht werden. Brigitte Schalhorn

Hurvitz SA et al. Combination of everolimus with trastuzumab plus paclitaxel as first-line treatment for patients with HER2-positve advanced breast cancer (BOLERO-1): a phase 3, randomised double blind, multicentre trial. Lancet Oncol. 2015;16(7):816-29. 